\title{
EL DATO, LA INFORMACIÓN, EL CONOCIMIENTO Y SU PRODUCTIVIDAD EN EMPRESAS DEL SECTOR PÚBLICO DE MEDELLÍN ${ }^{*}$
}

\author{
José Enrique Arias Pérez ${ }^{* *}$ \\ Carlos Andrés Aristizábal Botero**
}

Recibido: julio 19 de 2010

Aceptado: enero 20 de 2011

\section{RESUMEN}

El objetivo de este artículo es identificar el significado del dato, la información y el conocimiento para dos organizaciones del sector público de Medellín: EPM-UNE e ISA, ambas con trayectoria en el campo de la gestión del conocimiento, y determinar cuál es el tipo de relación existente entre estos conceptos y la productividad del conocimiento. La investigación es de corte cualitativo, se utilizó la entrevista semiestructurada para recolectar la información, se realizaron procesos de codificación abierta, axial y se construyeron esquemas para su análisis. En cuanto a los resultados se evidencia que en estas organizaciones, los significados de dato, información y conocimiento son cercanos a la postura constructivista, y cuando el conocimiento se convierte en información y la información en dato, ello no constituye una pérdida de valor, es decir, no compromete su productividad.

\section{PALABRAS CLAVE}

Gestión del conocimiento, dato, información, conocimiento, generación de valor, capital intelectual.

\section{CLASIFICACIÓN JEL} M10, M12, M15

\section{CONTENIDO}

Introducción; 1. Dato, información y conocimiento; 2. La productividad del conocimiento; 3. Metodología; 4. Resultados; 5. Conclusiones; Bibliografía

- Este artículo es producto de la investigación "Gerencia del conocimiento en el sector público de la ciudad de mMdellín: estudio de casos de prácticas actuales", financiada por el Centro de Investigaciones de la Fundación Universitaria Luis Amigó, sede Medellín, realizada por el grupo en Gestión de Organizaciones (GORAS), en el año 2008.

* Administrador de Empresas, Fundación Universitaria Luis Amigó, Medellín, Colombia; especialista en Gerencia de Sistemas y Tecnología, Universidad Pontificia Bolivariana, Medellín, Colombia; candidato a magíster en Gestión de Ciencia, Tecnología e Innovación, Universidad de Antioquia, Medellín, Colombia; docente Universidad de Antioquia (UdeA), Medellín, Colombia, docente Fundación Universitaria Luis Amigó (Funlam), Medellín, Colombia; investigador del grupo de investigación GESTOR, UdeA, y de GORAS, Funlam. Dirección: transversal 51ª N 67b 90. Tel: (57-4) 4487666 Ext. 9611. Correo electrónico: jearias@economicas.udea.edu.co; jose. ariaspe@amigo.edu.co.

** Sociólogo, Universidad de Antioquia, Medellín, Colombia; especialista en Ambientes de Aprendizaje Virtual, Universidad Minuto de Dios, Bogotá, Colombia; magíster en Educación y Desarrollo Humano, Universidad de Manizales, Manizales, Colombia; docente Universidad de Antioquia (UdeA), Medellín, Colombia, docente Fundación Universitaria Luís Amigó, Medellín, Colombia; coordinador del grupo de investigación GORAS. Dirección: transversal 51A Nº 67b 90. Tel: (57-4) 4487666 Ext. 9611 . Correo electrónico: carlos.aristizabalbo@amigo.edu.co.

Semestre Económico, volumen 14, № 28, pp. 95-110 • ISSN 0120-6346, enero-junio de 2011, Medellín, Colombia 95 


\section{DATA, INFORMATION, KNOWLEDGE AND ITS PRODUCTIVITY IN COMPANIES OF THE PUBLIC SECTOR OF MEDELLIN}

\section{ABSTRACT}

The objective of this article is to identify the data side, the information and the knowledge for two different organizations of Medellin's public sector, EPM-UNE and ISA. Both with vast experience in knowledge management and determine which is the existent type of relation between these concepts and the knowledge productivity. The research has a qualitative scope and semi structured interviews where performed for the data collection, opened codification and axial process where also held and schemes where built for their analysis. Concerning the results, it can be evidenced that these organizations, their data meanings, their information and knowledge are close to the constructive structure, and when this last one becomes information, and it's on data, it does not constitute a value loss, meaning by this, it does not jeopardize its productivity.

\section{KEY WORDS}

Knowledge management, data, information, knowledge, value generation, intellectual capital

\section{JEL CLASSIFICATION}

M10, M12, M15

\section{CONTENTS}

Introduction; 1. Data, information and knowledge; 2. Knowledge productivity; 3. Methodology; 4. Results; 5. Conclusions; Bibliography.

\section{O DADO, A INFORMAÇÃO, O CONHECIMENTO E SUA PRODUTIVIDADE NAS EMPRESAS DO SETOR PÚBLICO EM MEDELLÍN}

\section{RESUMO}

O objetivo deste artigo é identificar o dado, a informação e o conhecimento para duas organizações do setor público em Medellín, EPM, UNEM e ISA, as duas com trajetória no campo da gestão do conhecimento, e determinar qual é o tipo de relação existente entre estes conceptos e a produtividade do conhecimento. A pesquisa é de corte qualitativo, usou-se a pesquisa de opinião semiestruturada para recolher a informação, realizaram-se processos de codificação aberta, axial e construíram-se esquemas para sua analise. Quanto aos resultados, evidencia-se que em estas organizações, os significados do dado, da informação e dos conhecimentos são aproximados à postura construtivista, e quando este último converte-se em informação e despois em dado, não significa uma perdida de valor, é dizer não compromete sua produtividade.

\section{PALAVRAS-CHAVES}

Gestão do conhecimento, dado, informação, conhecimento, geração de valor, capital intelectual.

CLASSIFICAÇÃO JEL M10, M12, M15

\section{CONTEÚDO}

Introdução; 1. Dado, informação e conhecimento; 2. A produtividade do conhecimento; 3. Metodologia; 4. Resultados; 5. Conclusões; Bibliografia. 


\section{INTRODUCCIÓN}

La sociedad del conocimiento prioriza el desarrollo de las facultades intelectuales y la adquisición continua de nuevos saberes que tengan alguna injerencia en el actuar de las personas y en su modo de interpretar la realidad; ello es considerado como el activo intangible más importante de las organizaciones y como factor primario para la generación y sostenibilidad de las ventajas competitivas según Bueno (1998, p. 220), Drucker (1993, p. 146) y Safón (2000, p. 150).

Por ello, en la literatura diversos autores se han dado a la tarea de distinguir y desentrañar los elementos básicos que preceden la creación y adquisición de conocimiento por parte de los individuos, y destacan, en la mayoría de los casos, que este proceso comienza con la captura de los datos del medio externo e interno y su estructuración para la correspondiente transformación en información, la cual, a su vez, es potencialmente convertible en conocimiento al ser asimilada por las personas.

Sostienen los expertos que una organización que no lleve a cabo tal distinción pierde la oportunidad de capturar datos clave que al ser estructurados y puestos en consideración de los miembros de la organización, puedan redundar en la generación de valor, según Durant-Law y Byrne (2007, p. 6), bien sea incidiendo en la toma de decisiones o desencadenando procesos de innovación en los que se transformen ideas en bienes o servicios, se mejoren procesos o se diseñen soluciones de impacto social.

Colombia no es ajena a tal situación; en los últimos años, varias organizaciones del sector público han incursionado en la implementación de iniciativas que dinamizan los procesos de conversión del dato en información, y de ésta en conocimiento, sin embargo, se logra identificar que muchos de sus empleados carecen de los elementos epistemológicos que fundamentan estas distinciones y un gran número de personadas involucradas directamente con la prácticas de gestión de conocimiento tienen un bajo nivel de apropiación de estos conceptos (Bernal, Turriago y Sierra, 2010, p.35).

El peligro de tal desconocimiento estriba en que las organizaciones pueden eventualmente concebir el conocimiento como un objeto, volverlo equivalente al dato y la información, y de esta manera desligarlo de elementos culturales, de la realidad simbólica, de la interpretación, y terminar reduciéndolo a un tema meramente tecnológico, lo cual compromete su capacidad de generar valor que es denominada productividad del conocimiento (Durant-Law, 2004, p.42).

En consecuencia, este artículo busca poner en evidencia las concepciones de dato, información y conocimiento en dos organizaciones del sector público en Medellín, partiendo de dos enfoques: constructivista y representativo; asimismo, entender mediante el concepto de productividad (Durant-Law, 2004, p.42), las conversiones de cada uno éstos y sus efectos sobre la generación de valor.

De ahí que este artículo esté estructurado de la siguiente manera: en primer lugar, se hace un recorrido por el dato, la información y el conocimiento; luego, se desarrolla la concepción de productividad del conocimiento (Durant-Law, 2004, p. 43); posteriormente se precisan los aspectos metodológicos y se presentan los resultados y las conclusiones.

\section{DATO, INFORMACIÓN Y CONOCIMIENTO}

Con relación a dato, información y conocimiento prevalecen en la literatura de la gestión del conocimiento dos enfoques teóricos: constructivista y representativo. Según Salmador (2006, p. 28), ambos han sido ampliamente desarrollados en la última década y reconocidos como las corrientes de pensamiento que fundamentan teóricamente los actuales modelos de gestión de conocimiento.

Ackoff (1996, p. 30) fue de los primeros en plantear la transformación del dato en información y de ésta en conocimiento hasta llegar a la sabiduría; esta distinción originalmente se formuló para dar cuenta de las fases de los procesos de aprendizaje, 
sin embargo, paulatinamente ha sido retomada por los teóricos de la gestión del conocimiento como estrategia para familiarizar a los neófitos con los conceptos básicos de esta temática, introducirlos en la comprensión de aspectos más complejos de la misma, y focalizar la labor de las tecnologías de la información y la mente humana de acuerdo con Durant-Law (2004, p. 47), debido a que las primeras son de gran utilidad a la hora de capturar los datos y estructurarlos en información, mientras que la segunda es la única capaz de crear conocimiento.

En la literatura de la gestión del conocimiento, se logra identificar que los principales autores sólo mencionan el dato, la información y el conocimiento, porque se considera que la distinción entre este último y la sabiduría carece de unas sólidas bases epistemológicas según Durant-Law (2004, p. 26), por lo cual, el conocimiento es el techo de cualquier proceso de conversión.

\subsection{Enfoque constructivista}

Desde la perspectiva constructivista, dato, información y conocimiento son conceptos diferentes; éstos se conciben como una pirámide en la que están jerarquizados de menor a mayor y son prerrequisitos el uno del otro en términos de conversión según Salmador (2006, p. 30) y Alavi y Leidner (1999, p. 5). Tradicionalmente el dato ha sido definido como un símbolo que aún no ha sido interpretado de acuerdo con Spek y Spijkervet (1997, p. 20), una simple observación del estado del mundo según Davenport (1997, p. 45), o como un hecho bruto, simple y discreto tal como lo afirman Bhatt (2001, p. 72), Beveren (2002, p. 19), Davenport y Prusak (1998, p. 46) y Herder y otros (2003, p. 110).

Por su parte, la información es entendida por Davenport y Prusak (1998, p. 48) y Nonaka y Takeuchi (1995, p. 23) como conjunto de mensajes; mientras que para Bollinger y Smith (2001, p. 10) son datos procesados, organizados según Bhatt (2001, p. 72) o con significado de acuerdo con Spek y Spijkervet (1997, p. 21), necesarios para la creación de cono- cimiento, el cual, a su vez, es definido por Bhatt (2001, p. 73) y Herder y otros (2003, p. 110) como información con sentido, valiosa según Davenport (1997, p. 46), evaluada, validada, codificada tal como lo afirma Earl (2001, p. 221) o, en palabras de Nonaka y Takeuchi (1995, p. 23), un flujo de información anclado en las creencias y compromisos de su poseedor.

Se evidencia, con base en todo lo anterior, que la relación entre dato, información y conocimiento es lineal, secuencial y progresiva, y que el proceso de transformación consiste en añadirle algo al dato para que llegue a ser información, y luego añadirle algo a la información para que se convierta en conocimiento según Moteleb y Woodman (2007, p. 57), aunque de acuerdo con Lang (2001, p. 48) y Yahya y Goh (2002, p. 460), se debe subrayar que subyacen a cada concepto unas variantes contextuales, de uso, evaluación e interpretación que permiten concluir que dato, información y conocimiento son radicalmente diferentes y excluyentes entre sí.

\subsection{Enfoque representativo}

Para los autores del enfoque representativo, de la talla de Moteleb y Woodman (2007, p. 60), los planteamientos constructivistas son intuitivos y caen en la banalización tanto del conocimiento, que por deducción terminaría siendo un conjunto de hechos brutos, procesados y con sentido, como de los modelos de conocimiento al reducirlos a simples formas más elevadas de información; según Tuomi (2000, p. 109), esto hace presumir erróneamente que el conocimiento, cuando no resulta ser algo diferente del dato y la información, no debe representar nada nuevo ni interesante para los sistemas de gestión del conocimiento; de acuerdo con Fahey y Prusak (1998, p. 270), ello ha generado traumatismos en su diseño y funcionamiento.

Además, señalan que el enfoque constructivista se fundamenta en el empirismo aristotélico, al dar por sentado que el objeto del conocimiento es una realidad impersonal y exterior que debe ser estu- 
diada empíricamente para poder aprehender sus estructuras y estados; por ello no es de extrañar que la observación de hechos brutos, simples y discretos sea el único paso para la creación de conocimiento aunque en el pasado estas ideas fueron fuertemente criticadas por los filósofos del conocimiento como Bergson, James, Husserl, Heidegger, Mead, MerleauPonty y Polanyi según Tuomi (1999, p. 36).

Gráfico 1. La conversión conocimiento-informacióndato en doble vía

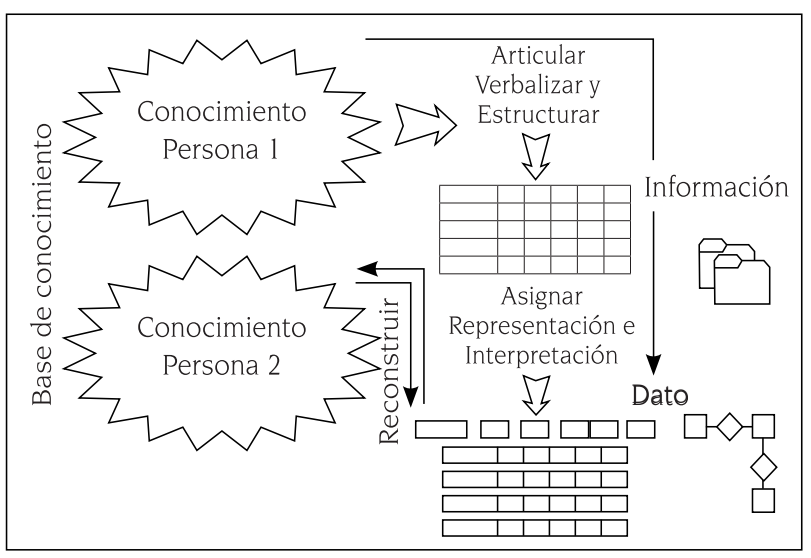

Fuente: Adaptado de Tuomi (2000, p. 14).

Los mismos autores de este enfoque, en búsqueda de una aproximación totalmente diferente, han invertido la pirámide y plantean que el dato bruto no existe y que el conocimiento precede a la información y al dato porque hasta el más elemental de los datos ha sido influenciado por los procesos de pensamiento y conocimiento que permitieron su identificación y recolección. Según Tuomi (2000, p. 110), desde esta corriente, el conocimiento es el punto de partida que, al ser articulado, verbalizado y estructurado, llega a ser información, la que posteriormente se torna dato una vez se atomiza y se le asigna una determinada representación e interpretación en una estructura semántica; se busca, entonces, que los individuos tengan la misma comprensión del dato y la información para lo cual deben primeramente compartir una base de conocimiento.
De no ser así, surgen dificultades cuando una segunda persona distinta a la que se tomó el trabajo de articular, estructurar su propio conocimiento y convertirlo en información y datos usando el lenguaje y los sistemas semánticos, se dispone a reconstruir el sentido potencial de los datos sin tener una base de conocimiento con la primera, en este caso, si ambas personas comparten un mundo o contexto que por lo general es tácito y no está representado en ningún sistema de información, la segunda podrá convertir el dato en información y luego en conocimiento.

\section{LA PRODUCTIVIDAD DEL CONOCIMIENTO}

Algunos autores han integrado el concepto de "la pirámide" de Ackoff (1996, p. 30) con "la espiral" de Nonaka y Takeuchi (1995, p. 48) en un concepto denominado por Durant-Law (2004, p. 42) como "el conducto del conocimiento", el cual es el pilar de la productividad, que se entiende como la generación de valor a partir de este recurso.

En este sentido, se plantea que el conocimiento llega a ser productivo cuando las organizaciones brindan unas condiciones de orden contextual que les permiten a los individuos capturar los datos y la información, movilizarlos a través del conducto hasta convertirse en conocimiento que pasa de tácito a explícito, y viceversa, tomando forma de espiral para generar valor, aunque también para este propósito debe evitarse que éste se degenere mediante los procesos de difusión y ósmosis en dato e información; este último planteamiento se constituye en un reconocimiento de la viabilidad de este otro tipo de conversión; a la inversa, ello concuerda con los postulados del enfoque representativo. (Gráfico 2)

El conducto cuenta con dos campos distintos: el dominio descriptivo y el predictivo; los datos y la información pertenecen al primero. En este marco los datos, según Davenport y Prusak (1998, p.46), se conciben como un conjunto de hechos objetivos, discretos que existen en forma simbólica y no han 
sido interpretados. En el conducto de conocimiento cuando los datos se enriquecen mediante la adición de contexto se convierten en información, es decir, datos con un mensaje, y por lo tanto, tienen receptor y remitente.

La información que se mueve hacia arriba en el conducto puede convertirse en conocimiento, al sumarle comprensión más acción; éste es, a la vez, descriptivo, predictivo y adaptativo, y es aplicable a muchas situaciones.

El conducto de conocimiento también tiene bucles de retroalimentación de conocimiento a información y a datos. Estos pueden ser llamados ósmosis y difusión según Boisot (1994, p. 190) que son conceptos cercanos el enfoque representativo.

La difusión, sinónimo de diseminación, aplica al conocimiento explícito que puede acrecentarse si los individuos le agregan comprensión, expe- riencia, y codifican esta sumatoria; caso contrario, el conocimiento se degeneraría en información o datos, aunque ambos pueden eventualmente transformarse en conocimiento tácito y explícito si son enriquecidos con el contexto y demás elementos.

La ósmosis se produce cuando es imposible codificar el conocimiento tácito, lo que resulta en una transferencia de intangibles de una persona a otra mediante la observación, la imitación, como en el caso del maestro y el aprendiz, lo cual ocurre en el terreno de lo descriptivo, de la información y los datos.

\section{METODOLOGÍA}

Para desarrollar la investigación se utilizó un enfoque metodológico de carácter cualitativo, donde primó la intención comprensiva, partiendo de los testimonios y narraciones de los sujetos de

Gráfico 2. El conducto de conocimiento

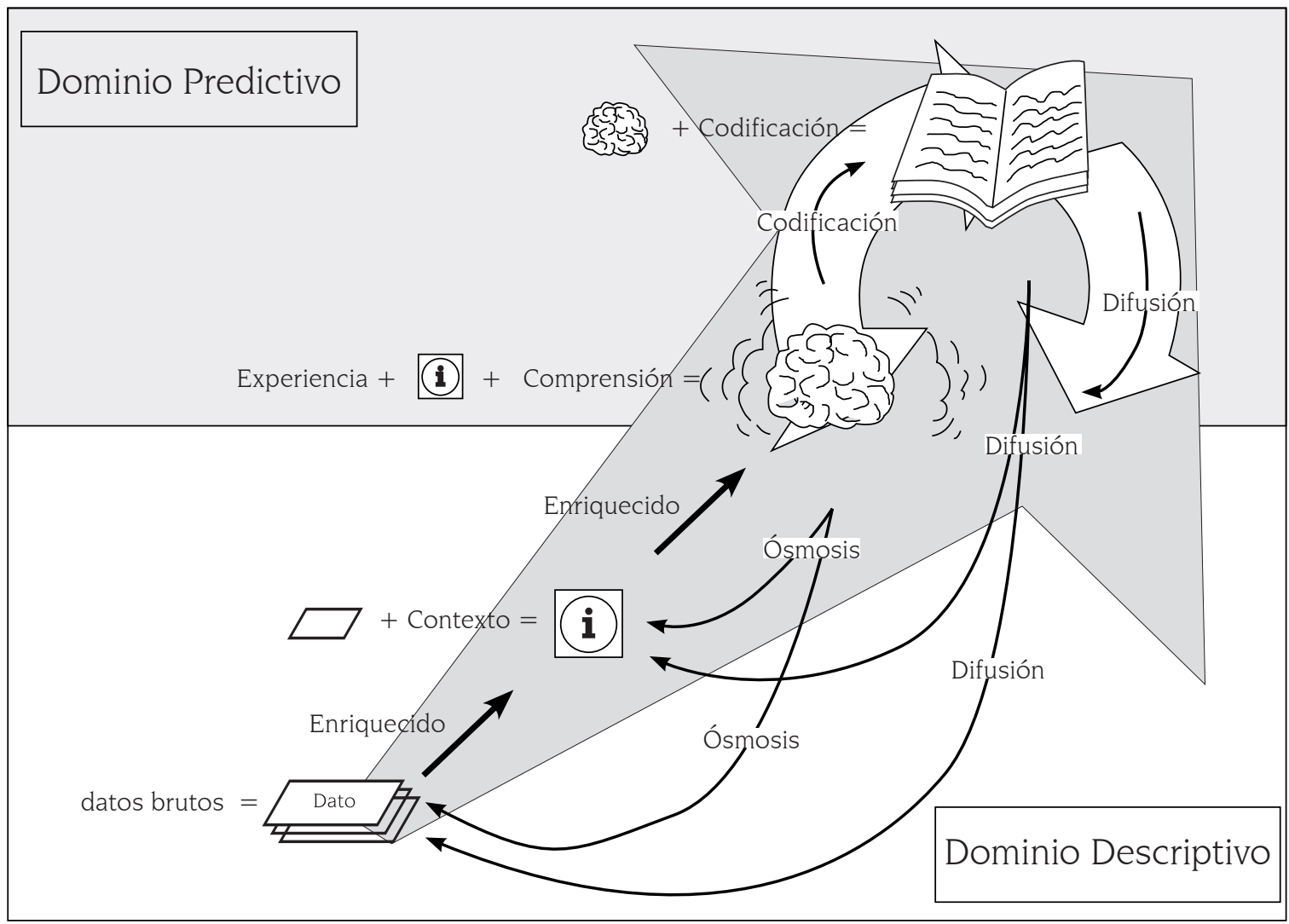

Fuente: Adaptado de Durant-Law (2004, p. 46) 
investigación, con el fin de capturar los significados alrededor de dato, información y conocimiento en un contexto organizacional.

Para la recolección de información, se diseñó un instrumento tipo entrevista semiestructurada (ver anexo A), con el propósito de lograr estimular la conversación sobre los tópicos propuesto; ésta permitió la transformación y reorientación de las preguntas según lo demandaba el curso de la conversación, así como la recuperación de opiniones y percepciones particulares de los entrevistados. Se realizaron en total siete entrevistas.

Para la selección de las empresas participantes en el estudio, se establecieron dos criterios de selección:

1. Ser una empresa del sector público de la ciudad de Medellín.

2. Haber implementado en algún momento estrategias y propuestas de gestión de conocimiento.

El trabajo de campo se realizó en EPM-UNE e ISA, y se entrevistaron 9 funcionarios clave, los cuales han gestionado el conocimiento desde los niveles operativos, tácticos y estratégicos, en las áreas de gestión humana, de tecnologías de la información y la comunicación, y en planeación, todos ellos por más de cinco años.

El análisis de información se llevó a cabo utilizando la estrategia de codificación de la teoría fundada, por lo cual se utilizó la codificación abierta y axial, para lo cual se tuvo el apoyo del programa Atlas ti1. En el proceso se tuvieron los siguientes momentos: primero, se redujeron las entrevistas a citas o fragmentos de información testimonial; segundo, se agruparon las citas en códigos abiertos correspondientes a unos códigos iniciales, con los cuales se buscó recuperar citas con similitudes, recurrencias y vínculos comunes. Posteriormente, se generaron los códigos axiales, los cuales recupe-

1 Atlas.ti versión 5.2, es un programa de fabricación alemana, que busca facilitar los proceso relacionados con el análisis cualitativo, éste permite la generación de códigos, la implementación de strategias de relación, la escritura de notas y memos de forma organizada y la generación de mapas. raron el sentido amplio de los códigos y permitieron identificar temas transversales al análisis.

En el proceso de codificación, se buscó recuperar las reflexiones alrededor de la información por medio de la escritura de memos, en los cuales se consignaron descripciones, aportes conceptuales, preguntas metodológicas, que posteriormente aportaron a la construcción de informes y artículos.

Con el fin de expresar la información de manera grafica, se diseñaron mapas conceptuales en los cuales se representaron las relaciones existentes entre los códigos y los testimonios, de tal manera que se lograra dar cuenta de categorías y subcategorías. Lo anterior demandó del grupo de trabajo mayor reflexión en torno a los elementos del mapa, desencadenando nuevos procesos de recodificación y generación de códigos que permitieran agrupar las nuevas consideraciones y recurrencias identificadas, así como generar hipótesis de trabajo que posibilitaran la descripción del fenómeno estudiado.

\section{RESULTADOS}

En las organizaciones del sector público se considera que la distinción entre el dato, la información y el conocimiento es pertinente y necesaria, especialmente en el área de gestión humana, y tecnología de la información y comunicaciones, puesto que de esta manera se brindan condiciones que propician la captura y estructuración de los datos, la salvaguarda de la información, y la comprensión acerca de lo que es el conocimiento.

En la empresa $\# 2^{2}$, se hace énfasis en deslindar los tres conceptos, buscando con ello crear conciencia sobre la necesidad de capturar y estructurar los datos entre los miembros de la organización, y de este modo convertirlos en información que luego pueda estar a disposición de todas las personas por medio de las tecnologías de la información (TI); porque es usual que las personas identifiquen

\footnotetext{
2 Por acuerdos de confidencialidad, los nombres de las empresas son sustituidos por las expresiones empresa \# 1 y empresa \# 2.
} 
datos clave del medio externo o interno pero no se den a la tarea de articularlos, y así se pierde una oportunidad de generar valor, de mitigar el riesgo en la toma de decisiones, aun cuando esto se lleve a cabo por otros individuos en otro extremo de la organización.

Entre tanto, en la empresa \#1, se identifica que la distinción es realizada principalmente por los ingenieros de sistemas, quienes habitualmente revisan el flujo del modelo de datos del sistema de información, con el propósito de afinar la manera tanto de capturar los datos del medio externo, como de estructurarlos para que se conviertan en información, y fluyan como tal a través del sistema. En este caso se asume que el dato es el insumo primario sobre el cual se trabaja y del que no se puede prescindir para la creación de conocimiento, además, las personas del sector público destacan que históricamente los ingenieros fueron los primeros en reflexionar sobre el particular, buscando precisar las diferencias entre el dato, la información y el conocimiento y determinar el rol y los límites de las TI en la gestión de estos recursos.

En este sentido, se considera que el papel que juegan las TI en la gestión del conocimiento consiste, por un lado, en realizar la captura y estructuración de los datos para que los individuos puedan crear conocimiento, y por otro, en evitar que se degrade el universo de información de la organización, lo cual requiere diseñar planes de continuidad de la información, definir políticas de seguridad para salvaguardar la información de las pérdidas provocadas por eventos catastróficos, en otras palabras, evitar que la organización pierda sus activos intangibles por el daño de los sistemas, discos duros, y demás medios de almacenamiento.

Asimismo, se evidencia que no sólo los responsables de los sistemas de información distinguen el dato, de la información y el conocimiento, sino también los expertos de la gestión del conocimiento, aquellos que han tenido una aproximación teórica al tema. Sin embargo, en los niveles operativos, entre aquéllos que participan en las distintas prácticas o metodologías como las comunidades de conocimiento, lecciones aprendidas, páginas amarillas, se desconoce la distinción; simplemente los individuos estructuran el dato en información y la interiorizan de manera natural, sin mayor conciencia de las distintas etapas de la conversión. Caso contrario sucede en la empresa \#2, donde se hacen esfuerzos por trazar la línea divisoria a lo largo y ancho de la pirámide organizacional.

Se evidencia, pues, un interés por acentuar la diferencia entre los tres conceptos, con el propósito de mostrar que la creación de conocimiento es un engranaje que depende de la ejecución de otras actividades que a simple vista pueden resultar irrelevantes como la captura y estructuración del dato en información, lo cual no debe ser una responsabilidad que recae exclusivamente sobre los hombros del área de tecnología e informática, sino que todas las personas por tener una base de conocimiento están en condiciones de identificar datos significativos para la organización.

\subsection{Significado y conversión del dato, la información y el conocimiento}

En cuanto al significado y conversión del dato, la información y el conocimiento, se observa que prima una postura cercana a la concepción constructivista, puesto que se toma el dato como punto de partida, al que luego se le añade algo para que llegue a ser información y posteriormente se procede de igual modo con esta última para convertirla en conocimiento.

En ese orden de ideas, en el sector público parece ser que se concibe el dato como una cifra carente de significado, o elemento mínimo, insumo básico, sustrato que luego llega a ser información cuando se le estructura, organiza y ordena dándole sentido; de igual manera, se da por hecho que la información se vuelve conocimiento cuando se alimenta de la experiencia y conduce a la acción o a la puesta en funcionamiento, proceso en el cual 
el individuo se apropia de la información, la analiza y la utiliza para interpretar e intervenir la realidad.

Una forma utilizada por un entrevistado para ilustrar dicho proceso es el caso del individuo que tiene en su agenda un sinnúmero de direcciones sin ninguna relación con otra variable, ello puede considerarse un simple dato; pero si a esas direcciones se les suman los nombres de las personas que allí residen esto se constituiría en información, es decir, un conjunto de datos estructurados y con sentido.

En cuanto a la conversión de la información en conocimiento la experiencia puede ser el detonante, lo cual puede ser ejemplificado de la siguiente manera: "en el caso de la señora del tinto que fue regañada por interrumpir una reunión importante, ella sabrá por esa experiencia que aun cuando se cumpla el horario para servir el tinto no podrá hacerlo sino hasta que finalice la reunión, eso es conocimiento" (Ríos, 2008).

O también puede ilustrarse todo el proceso de conversión de la siguiente manera: en primer lugar, cuando se busca mejorar un proceso lo primero que se hace es reunir un conjunto de datos y puntos de referencia como determinar quién sabe del tema, cuál es su ubicación, entre otros; luego, al diseñar y armar todo el proceso, los datos se convierten en información, y una vez se divulga, y se pone en funcionamiento, la información llega a ser conocimiento.

\section{2. ¿El conocimiento se gestiona o se facilita?}

De otra parte, cabe resaltar que los expertos en gestión de conocimiento del sector público consideran que no hay ninguna diferencia entre información y conocimiento explícito, porque ambos conceptos aluden a aquello que le pertenece a la organización y se puede expresar por medio de símbolos codificados, bien sea por medio del habla o la escritura de un texto o el diseño de un plano o esquema, de tal manera que otros puedan oírlo, percibirlo, entenderlo y llevarlo a la práctica.
Lo anterior sirve de fondo para desvelar la diferencia entre conocimiento explícito y tácito; en el sector público, se plantea que sólo este último puede considerarse como conocimiento, el otro es sólo información por las razones expuestas en el párrafo anterior, mientras que el segundo, a diferencia del primero, no se puede comunicar y es el que realmente permite interpretar la realidad y desencadenar acciones que en ocasiones no pueden ser explicadas racionalmente por el individuo que las ejecuta; ello suele evidenciarse en situaciones en las que el sujeto logra resolver algún problema pero es incapaz de señalar cómo lo hizo. Pero, además, se considera que otra diferencia sustancial entre ambos es que sólo el tácito puede generar valor para la organización.

Dada esta diferencia entre los dos tipos de conocimiento, los expertos se han preguntado si realmente es procedente hablar de gestión del conocimiento tácito; en este sentido, apoyados en la experiencia y en algunos planteamientos teóricos, han llegado a la conclusión de que esto no es posible; en lugar de ello se puede facilitar el que en realidad se puede gestionar: el explícito.

En el sector público, facilitar el conocimiento tácito tiene una connotación humana y se traduce en cierto tipo de liderazgo basado en la figura del jefe facilitador o líder servidor que, sin coacciones, logra motivar e impulsar al empleado a compartir y utilizar el conocimiento para generar valor. Ello, en la práctica, ocurre cuando el jefe le dice al experto: "vos sos experto en tal tema por qué no te preparás un cursito para que lo multipliquemos aquí (...), entonces, como yo soy un experto en tal tema, yo puedo decir sí o no, yo realmente no me veo como profesor; simplemente, déjeme trabajar; yo no doy cursos" (Parra, 2008). Por lo tanto, la función del facilitador se resume en "poner todo pulpito para que la gente trabaje (...), y aplique, use y potencie su conocimiento" (Parra, 2008).

Gestionar el conocimiento explícito tiene una connotación de orden tecnológico, normativo, y de movilización de recursos. Esto puede evidenciarse,

Semestre Económico, volumen 14, № 28, pp. 95-110 • ISSN 0120-6346, enero-junio de 2011, Medellín, Colombia 103 
por un lado, en las actividades que realiza la biblioteca o cualquier sistema de archivo apoyados en las TI, tales como captura, almacenamiento y difusión de los datos e información del medio externo e interno; por otro, en la promulgación de decretos que obliguen a los empleados a documentar para explicitar el conocimiento, como los decretos de capacitación según los cuales todas las personas que participen de cualquier evento de esta naturaleza deben redactar un informe sobre su aprendizaje y experiencias.

En el sector público el conocimiento se gestiona y facilita como respuesta a dos grandes situaciones que se presentan en esta organización: la concentración de conocimiento y la amplia brecha entre lo que se debe saber y lo que se sabe.

Con respecto a lo primero, existe entre los directivos el interés por desmantelar todas las barreras que obstruyen la diseminación del conocimiento, porque han identificado que hay un alto número de personas renuentes a compartir su conocimiento por no ver con agrado esta actividad y por considerar que al hacerlo ponen en riesgo su participación en diferentes proyectos, al dejar de ser imprescindibles. Por ende, ello se ha constituido en una debilidad que ha generado diversos contratiempos en el curso normal de los procesos, porque en ocasiones se presentan daños en las plataformas tecnológicas y las personas con el conocimiento para solucionar cierto tipo de contingencias no se encuentran a disposición de la organización en un determinado momento.

En este sentido algo similar ocurre con el manejo de algunos equipos tecnológicos de diversas características heredados de las empresas fusionadas; en la mayoría de los casos las personas que se incorporaron a la empresa \#1 venían especializadas en el funcionamiento de los mismos; sin embargo, las directivas de la organización están interesadas en que "Jorge aprenda lo que sabe Gabriel, lo que sabe Mario, todos necesitamos trabajar en todo, porque si en un momento determinado falta Mario ahí esta Jorge" (Ruiz, 2008).
En cuanto a la brecha de conocimiento, en las empresas del sector público se trabaja arduamente en definir lo que deben saber los empleados de la organización. Como es sabido, por la fusión, en esta organización hay distintas maneras de prestar un mismo servicio según la procedencia del funcionario. Sin embargo, las directivas están promoviendo la unificación de los procedimientos y la reestructuración de varios de los procesos para que estos respondan a las necesidades actuales de los clientes, y no al parecer personal de los empleados; de este modo se está configurando un ideal de conocimiento que obliga a los funcionarios a abandonar las antiguas prácticas y aprender el nuevo saber hacer.

\subsection{La productividad del conocimiento}

En la empresa \#1, se han evidenciado distintos procesos de conversión del dato a la información y de ésta al conocimiento, como en el caso del área de tecnología de información y comunicaciones donde capturan los datos, los estructuran para transformarlos en información, de la cual los individuos se apropian hasta llegar al conocimiento que les permite actuar e interpretar la realidad; o en el caso de la funcionaria que considera que la información pasa a ser conocimiento sólo cuando cualquier actividad o proceso pasa del papel a la ejecución y divulgación.

Además, hay experiencias concretas de conversión en las que se vislumbra la generación de valor. Un ejemplo es la venta del servicio de televisión interactiva, proceso que requiere como punto de partida la captura de datos clave, tales como costos del producto, ciudad y público objetivo, crecimiento de la población, entre otros; éstos, al ser estructurados, se convierten en información que logra transmitir un sentido o significado al colocar los datos en contexto, en este caso, da pistas sobre la viabilidad de este servicio en un determinado mercado. La organización combina los datos como crecimiento de la población en determinada ciudad, 
y de este modo, obtiene un material que entraña un sentido, un contexto y que puede ser objeto de análisis y soporte en la toma de decisiones.

De otra parte, en el sector público tal como se señaló, no hay distinción entre conocimiento explícito e información, por ende, cuando el conocimiento se convierte en información ello no implica que se esté degradando, sino que está adoptando una forma en la que puede transferirse para que otro sujeto logre interiorizarla, y convertirla en conocimiento con base en sus intereses, valores y creencias; en palabras del entrevistado,
... yo estoy hablando en este momento de ciertas cosas de conocimiento y ustedes pueden estar adquiriéndolo o interiorizándolo de forma diferente, de acuerdo a lo que cada uno esta buscando (...), en este momento estamos transfiriendo esa información que son conocimientos míos que se convierten en una información para ustedes y apenas lo interioricen y lo incorporen frente a lo que ustedes piensan se va a transformar en conocimiento (Vanegas, 2008).

Sumado a lo anterior, en el sector público se considera que la conversión de la información en

Gráfico 3. Dato, información y conocimiento en el sector público

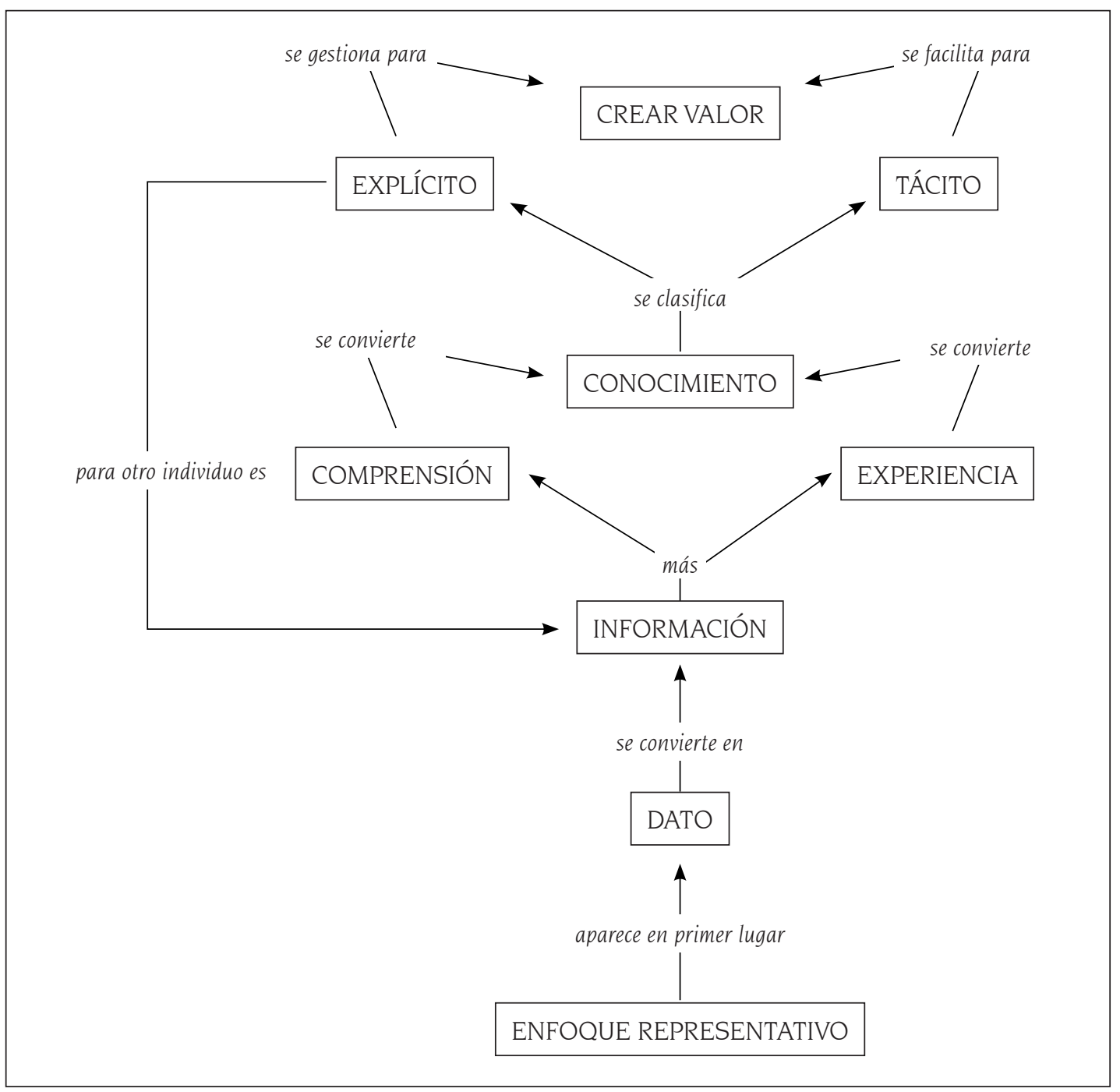

Fuente: Elaboración propia

Semestre Económico, volumen 14, № 28, pp. 95-110 • ISSN 0120-6346, enero-junio de 2011, Medellín, Colombia 105 
dato tampoco está asociada con la degradación que conlleva a la pérdida de la productividad, por el contrario, se asume que la creación de conocimiento es un proceso que en su etapa de maduración termina lanzando datos clave o puede decirse también que el conocimiento puede representarse en este formato, como en el caso, de la construcción de modelos financieros que son el resultado de la conversión del dato en información y de ésta en conocimiento, que una vez se pone en funcionamiento sirve para arrojar datos.

Aunque algunos expertos de la gestión del conocimiento consideran inviable que la información se transforme en dato debido a la imposibilidad de desarticular un conjunto de datos estructurados, de todas maneras en el sector público se observa que la transformación del dato en información y de ésta en conocimiento es de doble vía, porque es una espiral en la que después de llegar al conocimiento, se obtiene información y datos que sirven de insumos para un nuevos procesos de conversión; algunos plantean que es como una telaraña

... del dato pasamos a la información, de la información al conocimiento y luego eso se devuelve (...), con ese conocimiento podes generar datos o podes dar como información, o sea, eso es como generar una araña, pues, una telaraña donde se abre ese conocimiento, se generan más datos, se retroalimenta de información y te manda otra información al sistema (...), siempre estas abriendo muchas más fuentes de conocimiento" (Vanegas, 2008).

Sin embargo, hay conciencia de que el conocimiento se degenera dejando de ser productivo al no generar valor; una de las razones, obedece a que las personas aun cuando han interiorizado la información tienen dificultades para llevarla a la acción por no estar desempeñándose en un puesto de trabajo que brinde las condiciones adecuadas para ello; por eso "cuando menos lo pienso, pasan los años y ya se me olvido lo que yo tanto sabía" (Ruiz, 2008).
También se tiene la percepción de que el conocimiento se degenera cuando lo que está funcionando bien, sea un proceso o una actividad, deja de estarlo y pierde divulgación, tal como sucede con la gestión del conocimiento en la empresa \#1 que aun cuando fue vigorosa en ORBITEL y EPM, a raíz de la fusión ha sido relegada a un segundo plano en esta nueva organización.

Igualmente, hay degradación cuando la organización no logra articular la información y el conocimiento individual con el organizacional, como en el caso del funcionario que tiene en su cubículo una serie de documentos de referencia que nutren día a día su trabajo; si eventualmente éste se retirara de la organización y los llevara consigo, el universo de información de la organización sufriría pérdida y se degradaría. Algo similar sucede cuando los repositorios de conocimiento, discos duros, memorias, sufren algún daño y los activos intangibles allí depositados se malogran.

Del mismo modo, las deficiencias en la gestión del conocimiento contribuyen a la degeneración del mismo; tal como sucede cuando las personas llevan mucho tiempo laborando en la organización están a las puertas de su jubilación y no se pone en marcha ninguna práctica que busque capturar el conocimiento que esta persona ha acumulado a lo largo de tantos años; si ello es así, la organización pierde gran cantidad de activos intangibles, relaciones y demás.

Otro caso típico que le resta productividad al conocimiento son las demoras en la toma de decisiones, lo cual es frecuente en organizaciones del sector público; a pesar de que internamente se llevó a cabo un proceso de generación de conocimiento que ha reducido la incertidumbre que circunda la decisión, aparece una serie de barreras organizacionales, especialmente de carácter normativo que terminan retrasando su toma en el tiempo previsto, sin embargo, son los cambios repentinos en el mercado de las telecomunicaciones los que terminan erosionando el valor a generar al dar ese paso. 
También el entorno cumple otro papel en lo concerniente a la degeneración, porque los cambios tecnológicos tornan obsoletos los conocimientos con los que operan los empleados de la organización. Por tal razón, en ocasiones las directivas se ven abocadas a poner en marcha planes de capacitación para que los funcionarios "pierdan ese conocimiento, pero a la vez que les sirva de aprendizaje para uno nuevo" (Ruiz, 2008).

\section{CONCLUSIONES}

Aun cuando se considera importante la distinción entre el dato, la información y el conocimiento, en estas organizaciones se asume que este asunto es del resorte del área de tecnología de la información y comunicaciones, lo que evidencia que ha sido poca la trascendencia que se le ha dado en el área humana que es la encargada de propiciar de manera directa los procesos de creación de conocimiento.

Además, se observa que en estas empresas del sector público, el dato, la información y el conocimiento se conciben desde el enfoque constructivista, y la concepción que se tiene del conocimiento es cercana, por un lado, a la acción, y por otro, al aumento en la capacidad interpretativa de la realidad. En lo concerniente a la productividad del conocimiento se evidencia que el conocimiento cuando se convierte en información y ésta en dato no constituye una pérdida de valor.

En lo concerniente a la praxis de la gestión del conocimiento, una de las contribuciones de este estudio radica en señalar la importancia de recalcar en los miembros de la organización vinculados a los procesos de creación de conocimiento, especialmente en aquéllos que no son expertos en la gestión del conocimiento ni ingenieros, la importancia de identificar, capturar y explotar el dato como punto de partida para la productividad del conocimiento, dándole relevancia para no desperdiciar la más mínima oportunidad de generar valor a partir del mismo, para lo cual, una de las alternativas es comenzar a concebirlo como conocimiento en un formato diferente, lo cual implica el tránsito del enfoque constructivista al representativo. De igual modo ocurre con la información.

Adicionalmente, este estudio pone de manifiesto la distinción que se está dando entre facilitar y gestionar el conocimiento en el sector público, con miras a precisar y clasificar las estrategias que permitan un mejor aprovechamiento del tipo tácito o explícito. Cabe destacar la utilización de normas para forzar la codificación del conocimiento por parte de los miembros de estas organizaciones hasta volver esta acción algo habitual.

De otra parte, se resalta la importancia del cambio de enfoque, del constructivista al representativo, como fórmula para evitar que el conocimiento al convertirse en información o dato pierda su capacidad de generar valor; lo que sí merece la atención y el monitoreo de las organizaciones y los encargados de la gestión del conocimiento es el conjunto de factores que lo degeneran, a saber, los cambios en el entorno especialmente los tecnológicos, las dificultades para pasar de la comprensión a la acción, la tendencia a reducir la divulgación y dejar fuera de funcionamiento prácticas que generan valor, y la lentitud en el proceso de toma de decisiones.

\section{BIBLIOGRAFÍA}

Ackoff, R. (1996). On learning and systems that facilitate it. En: Center for quality of management journal, Vol. 5, No 2, p. 27-35.

Alavi, M. y Leidner, D. (1999). Knowledge Management Systems: Issues, Challenges, and Benefits. En: Communications of the Association for Information Systems, Vol.1, No. 7, p. 1-37.

Bernal, C; Turriago, A. y Sierra, H. (2010). Aproximación a la medición de la gestión del conocimiento empresarial. En: Revista Ad-minister, Vol. 1, No. 16, p. 30-49.

Beveren J. V. (2002). A model of Knowledge Acquisition that Refocuses Knowledge Management. En: Journal of Knowledge Management, Vol. 6, No. 1, p. 18-22.

Bhatt, G. (2001). Knowledge management in organisations: Examining the interaction between technolo-

Semestre Económico, volumen 14, № 28, pp. 95-110 • ISSN 0120-6346, enero-junio de 2011, Medellín, Colombia 107 
gies, techniques, and people. En: Journal of Knowledge Management, Vol. 5, No. 1, p. 68-75.

Bollinger A. y Smith R. (2001) Managing organisational knowledge as a strategic asset. En: Journal of Knowledge Management, Vol. 5, No. 1, p. 8-18.

Boisot, M. (1994). Information and organizations: The manager as anthropologist. London: Harper Collins. 208p.

Bueno, E. (1998). El capital intangible como clave estratégica en la competencia actual. En: Boletín de Estudios Económicos, Vol. 53, No 164, p. 207-229.

Davenport, T. (1997). Information Ecology: Mastering the information and knowledge enviroment. New York: Oxford University Press. 272p.

Davenport, T. y Prusak, L. (1998). Working Knowledge: How organisations manage what they know. USA: Harvard Business School Press, 224p.

Durant-Law, G. (2004). Tardis: a journey through an enterprise knowledge space. [En línea] University of Canberra, Canberra Australia. Disponible en: http://www.durantlaw. info/sites/durantlaw.info/files/TARDIS Final Presentation.pdf, 113p.

Durant-Law, G. y Byrne P. (2007). The Tardis Knowledge Productivity System. Australian Government. [En línea] Deparment of Defence, Camberra Australia, Disponible en: http://www.durantlaw.info/sites/durantlaw. info/files/TARDIS\%20Manual.pdf, 134p.

Drucker, P. (1993). Post-capitalist society. Oxford: Butterworth-Heinemann, 218p.

Earl, M. (2001). Knowledge Management Strategies: toward a taxonomy. En: Journal of Management Information Systems, Vol.18, No.1, p. 215-233.

Fahey, L. y Prusak, L. (1998). The eleven deadliest sins of knowledge Management. En: California Management Review, Vol. 40, No 3, p. 265-276.

Herder P; Veeneman W; Buitenhuis M. y Schaller A. (2003). Follow the rainbow: a knowledge management framework for new product introduction. Journal of Knowledge Management, Vol. 7, No. 3, p. 105-115.
Lang J. (2001). Managerial concerns in knowledge management. En: Journal of Knowledge Management, Vol. 5, No 1, p. 43-57.

Moteleb A. y Woodman M. (2007). Notions of Knowledge Management Systems: a Gap Analysis. The Electronic Journal of Knowledge Management Vol. 5, No 1, p. 55-62.

Nonaka, I. y Takeuchi, H. (1995). The Knowledge-Creating Company, Oxford: Oxford University Press, 304p.

Parra, I. (2008). EPM-UNE. Entrevista inédita, Medellín, 05 de septiembre.

Ríos, G. (2008). EPM-UNE. Entrevista inédita, Medellín, 12 de septiembre.

Ruíz, L. (2008). EPM-UNE. Entrevista inédita, Medellín, 12 de septiembre

Safón, V. (2000). La flexibilidad de la empresa: Teoría, cuantificación y práctica. Valencia: Editorial Promolibro, 221p.

Salmador, M (2006). Raíces epistemológicas del conocimiento organizativo, estudio de sus dimensiones. En Economía Industrial, Vol. 1, No. 357, p. 27-57.

Spek, R y Spijkervet, A. (1997). Knowledge management: Dealing Intelligently with knowledge. Utrecht: Kenniscentrum CIBIT. 25p.

Vanegas, C. (2008). EPM-UNE. Entrevista inédita, Medellín, 05 de septiembre.

Yahya S. Y Goh W. (2002). Managing human resources toward achieving knowledge management. Journal of Knowledge Management, Vol. 6, No. 5, p. 457-468.

Tuomi, I. (1999). Corporate knowledge: Theory and practice of intelligent organizations. Helsinki: Metaxis. 453p.

Tuomi, I. (2000). Data is more than Knowledge: Implications of the reversed knowledge hierarchy for knowledge managment and organizational memory. Journal of Management Information Systems, Vol. 16, No. 3, p. 103-116. 


\section{Anexo A. Guía de Preguntas de la Entrevista Semiestructurada}

1. ¿Qué entiende usted y la organización por dato, información y conocimiento?

2. ¿Es importante hacer tal distinción?

3. ¿Cuál es la diferencia entre información y conocimiento explícito?

4. ¿Cuáles son los criterios para articular el conocimiento creado por los grupos e individuos con el conocimiento organizacional?

5. ¿Cuáles son los criterios para falsear el conocimiento creado por los grupos e individuos?

6. ¿Cuáles son los criterios que usted como individuo usa para aceptar o rechazar el conocimiento que es creado por un compañero o grupo de trabajo?

7. ¿Cuáles son los criterios que usted como individuos usa para aceptar o rechazar el conocimiento que es traído del medio externo de la organización?

8. ¿De qué manera la organización incorpora los nuevos conocimientos al conocimiento organizacional?

9. ¿De qué manera usted como individuo convierte los datos y la información en conocimiento?

10. ¿Cómo la organización apoya este proceso de conversión dato-información en conocimiento?

11. ¿De qué manera convierte su conocimiento tácito en explícito, y viceversa?

12. ¿De qué manera usted como individuo evita que el conocimiento se convierta en información y datos?

13. ¿Cómo la organización evita que el conocimiento se degenere en información y datos?

14. ¿El ser una empresa del sector público dificulta o facilita la conversión dato-Información en conocimiento?

15. ¿El ser una empresa del sector público dificulta o facilita la conversión de conocimiento tácito en explícito y viceversa?

16. ¿El ser una empresa del sector pública propicia la degeneración del conocimiento en información y dato?

Semestre Económico, volumen 14, № 28, pp. 95-110 • ISSN 0120-6346, enero-junio de 2011, Medellín, Colombia 109 